\title{
Tratamiento sintomático en migraña. Fármacos utilizados y variables relacionadas. Resultados de la encuesta europea sobre trabajo y migraña
}

\section{Symptomatic treatment for migraña. Drugs used and related variables. Results of the european survey on work and migraine}

M. T. Vicente-Herrero ${ }^{1}$, M. V. Ramírez Iñiguez de la Torre'1, E. Ruiz de la Torre ${ }^{2}$ y L. Reinoso Barbero ${ }^{3}$

${ }^{1}$ Grupo de Trabajo de Guías y Protocolos Asociación Española de Especialistas en Medicina del Trabajo. España. ${ }^{2}$ Presidenta de la European Migraine and Headache Alliance. ${ }^{3}$ Presidente de la Asociación Española de Especialistas en Medicina del Trabajo. España

\section{ABSTRACT}

Goals: To know the use of migraine crisis symptomatic treatments in different countries of Europe and the differences observed depending on social and demographic variables.

Material and methods: Cross-sectional observational study by anonymous web survey to 3,342 patients from Spain, Italy, France, Portugal, Ireland, United Kingdom, Germany and other countries of the European Union. Study variables: age, gender, country, type of location, level of studies and rural or urban area. The symptomatic treatments that are collected are: simple analgesics, non-steroidal anti-inflammatory drugs, triptans, other treatments, several treatments, without treatment, ignorance of symptomatic treatment.

Results: For simple analgesics, the largest consumers are between 41-60 years ( $p<0.0001)$. Spain and Germany are the countries with the highest use $[\mathrm{p}<$ 0.0001 ). For the anti-inflammatories, the greatest use is between 21-60 years ( $p<0.0001$ ). Spain, Italy and Germany are the countries that make the most use of them $(p<0.0001)$ and higher consumption in patients with higher education $(\mathrm{p}<0.003)$.

Triptans use shows a relationship with age and gender, greater use between 21-60 years ( $p<0.0001$ ) and in women $(p<0.0001)$. By countries, the highest

\section{RESUMEN}

Objetivos: Conocer el uso de tratamientos sintomáticos para las crisis de migraña en distintos países de Europa y las diferencias que se observan en función de variables sociales y demográficas.

Material y métodos: Estudio observacional transversal mediante encuesta anónima vía web a 3342 pacientes de España, Italia, Francia, Portugal, Irlanda, Reino Unido, Alemania y otros países de la Unión Europea. Variables de estudio: edad género, país, tipo de localidad, nivel de estudios y ámbito rural o urbano. Los tratamientos sintomáticos que se recogen son: analgésicos simples, antinflamatorios no esteroideos, triptanes, otros tratamientos, varios tratamientos, sin tratamiento, desconocimiento de tratamiento sintomático.

Resultados: En analgésicos simples los mayores consumidores están entre 41-60 años ( $p<0,0001$ ). España y Alemania son los países con mayor uso ( $p<0,0001$ ). El cuanto a los antinflamatorios el mayor uso se da entre 21-60 años ( $p<0,0001)$. España, Italia y Alemania son los países que mayor uso hacen de ellos ( $p<0,0001$ ) y mayor consumo en pacientes con estudios superiores ( $p<0,003$ ).

El uso de triptanes muestra relación con la edad y el género, mayor uso entre 21-60 años ( $p<0,0001$ ) y en mujeres ( $p<0,0001)$. Por países, el mayor consumo
Vicente-Herrero MT, Ramírez lñiguez de la Torre MV, Ruiz de la Torre E, Reinoso Barbero L. Tratamiento sintomático en migraña. Fármacos utilizados y variables relacionadas. Resultados de la encuesta europea sobre trabajo y migraña. Rev Soc Esp Dolor. 2020;27(3):178-191
Recibido: 15-05-2019

Aceptado: 15-05-2020

Correspondencia: M. ${ }^{\text {a }}$ Teófila Vicente-Herrero mtvh@ono.com/grupo.gimt@gmail.com 
consumption is in Germany, the rest of the European Union countries, Spain and the United Kingdom ( $p<$ 0.0001 ). Greater consumption in patients living in cities with more than 500 inhabitants $(p<0.010)$ and with medium or higher studies $(p<0.0001)$. In the group of middle and upper studies, we find, by contrast, the highest percentage of patients who do not know what a symptomatic treatment is $(p<0.0001)$.

Conclusions: The result varies according each country greatly and they are modified depending on different social and demographic conditions, being the age, gender, rural or urban environment and cultural level the factors that most affect the use of each symptomatic medication for the migraine crisis.

Key words: Migraine, symptomatic treatment, pain, occupational medicine, occupational health, survey.

\section{INTRODUCCIÓN}

La migraña es una enfermedad con elevada prevalencia global y discapacitante, siendo aceptado que no se diagnostica ni se trata adecuadamente. Así lo muestran los resultados del estudio "Eurolight" realizado en 10 países europeos (Alemania, Italia, Lituania, Luxemburgo, Países Bajos, España, Austria, Francia, Reino Unido e Irlanda) y con 9247 pacientes participantes. Según se refleja en este trabajo, en los países europeos ricos, muy pocas personas con migraña consultan a los médicos y los medicamentos específicos para la migraña se usan de manera inadecuada, incluso entre los que sí lo hacen, lo que permite concluir que es necesario mejorar la atención de las personas con dolor de cabeza y los aspectos formativos en los responsables de la atención médica y entre los pacientes [1].

En esta línea se pronuncia también el estudio "My Migraine Voice", realizado en 31 países de América del Norte y del Sur, Europa, Medio Oriente y África del Norte y la región de Asia y el Pacífico, con participación de 11.266 personas y dirigido a comprender la carga total y el impacto de la enfermedad cuando las crisis se prolongan más de 4 días al mes. Los resultados de este estudio sugieren que los tratamientos correctamente indicados y usados, y los avances de desarrollo futuros abordarian las necesidades existentes actualmente y permitir que las personas con migraña puedan maximizar su contribución a la sociedad [?].

En Europa, el impacto social y económico de la migraña se relaciona con la duración de las crisis y su inadecuado control, lo que conlleva repercusión en la calidad de vida, la pérdida de productividad laboral y el elevado uso de recursos sanitarios [3]. A esto se añade el hecho de que muchos pacientes con migraña no consultan a un médico, o no logran un alivio adecuado después de consultar como consecuencia de un tratamiento insuficiente o no adecuado, y sigue habiendo una necesidad no satisfecha en el cuidado de la migraña (4).

Es objetivo de este trabajo conocer el uso de tratamientos sintomáticos para las crisis de migraña en es en Alemania, resto de países de Unión Europea, España y Reino Unido ( $p<0,0001$ ). Mayor consumo en pacientes que viven en ciudades de más de 500 habitantes $(p<0,010)$ y con estudios medios o superiores ( $p<0,0001$ ). En el grupo de estudios medios y superiores se encuentra por contraste el mayor porcentaje de pacientes que desconocen qué es un tratamiento sintomático ( $p<0,0001)$.

Conclusiones: Existe una gran variabilidad de los resultados en cada país y se modifican en función de distintas condiciones sociales y demográficas, siendo la edad, el género, el ámbito rural o urbano y el nivel cultural los factores que más condicionan el uso de cada medicación sintomática para las crisis de migraña.

Palabras clave: Migraña, tratamiento sintomático, dolor, medicina del trabajo, salud laboral, encuesta.

distintos países de Europa y las diferencias que se observan en función de las diferentes condiciones sociales y demográficas, de forma que permita plantear en el futuro actuaciones más eficaces y orientadas en base a los resultados obtenidos.

\section{MATERIAL Y MÉTODOS}

Estudio observacional transversal a partir de una encuesta anónima vía web ubicada en la página de la European Migraine and Headache Alliance (EMHA) y avalada científicamente por la asociación española de especialistas en medicina del trabajo (AEEMT) a 3342 pacientes de España, Italia, Francia, Portugal, Irlanda, Reino Unido, Alemania y otros países de la Unión Europea no incluidos entre los anteriores. Se consideran criterios de inclusión tener un diagnóstico previo de migraña, estar trabajando en el momento de la encuesta o haberlo estado en el año previo, y participación voluntaria. Se recogen datos desde septiembre de 2018 hasta enero de 2019. La encuesta consta de 32 cuestiones con respuesta múltiple (Anexo 1).

A partir del descriptivo inicial se analizan de forma específica los tratamientos sintomáticos utilizados para el tratamiento de las crisis en función de una serie de variables sociodemográficas: edad (menos de 20 años, entre 21-40, entre 41-60, más de 61), género (hombre, mujer], lugar de residencia [España, Italia, Francia, Portugal, Irlanda, Reino Unido, Alemania, otro país de la UE), tipo de localidad donde reside (hasta 500 habitantes, desde 500-10.000 habitantes, desde 10.000-250.000 habitantes, desde 250.000-1 millón de habitantes, más de un millón de habitantes], nivel de estudios (elementales, medios, superiores], ámbito en el que vive (rural [pueblo], urbano [capital]).

Los tratamientos sintomáticos utilizados vienen definidos por la pregunta 13 del cuestionario (Tratamiento para el dolor cuando tiene crisis de migraña: con analgésicos simples, con antinflamatorios no esteroideos (AINE), con triptanes, con otros tratamientos sintomá- 
ticos, con varios tratamientos sintomáticos no nombrados anteriormente, no llevo tratamiento sintomático, no sé qué es un tratamiento sintomático].

Se ha realizado un análisis bivariante para cada uno de los tipos de tratamientos sintomáticos utilizados según cada uno de los parámetros sociodemográficos.

Se presentan tablas de contingencia mostrando la frecuencia absoluta (N) y el porcentaje (\%) para cada cruce de variables. Según la naturaleza de las variables del cuestionario (variables categóricas) se ha utilizado el test Chi cuadrado o test exacto de Fisher para analizar la posible relación entre las características de la migraña y las variables sociodemográficas.

Dado que la pregunta 13 tiene respuestas múltiples, el análisis de los datos se ha realizado de forma independiente para cada una de las posibles respuestas.

\section{RESULTADOS}

Las características sociodemográficas de la población que ha respondido a la encuesta se muestran en la Ta- bla I e indican una heterogénea distribución por países, correspondiendo el mayor porcentaje de respuestas a España y Alemania. El 85,13\% de los encuestados se encuadran en el bloque de edad media y son mayoritariamente mujeres (90\%). Los participantes residen principalmente en ámbito urbano (68,63 \%), en ciudades de medio-gran tamaño (el $35 \%$ en localidades de más de 250.000 habitantes y el 72,5\% en localidades de más de 10.000 habitantes], son trabajadores cualificados (el $69 \%$ con estudios superiores y el $27 \%$ con estudios medios] y reciben un apoyo moderado de su entorno durante las crisis de migraña $[44,06 \%)$.

Los resultados globales del uso de los diferentes tratamientos sintomáticos en las crisis de migraña y su relación porcentual con las distintas variables sociodemográficas estudiadas se muestran en la Tabla II.

Cuando se diferencian cada uno de los tipos de tratamientos sintomáticos en relación con las variables estudiadas, y considerando solo aquellos resultados con significación estadística vemos que:

- El grupo comprendido entre los 41-60 años es el que mayor uso hace de analgésicos simples,

TABLA I

CARACTERÍSTICAS SOCIODEMOGRÁFICAS DE LA POBLACIÓN ENCUESTADA

\begin{tabular}{|c|c|c|c|}
\hline \multicolumn{2}{|r|}{ Variable } & $\%$ & $n$ \\
\hline \multirow{4}{*}{ Edad } & Menos de 20 años & $11,79 \%$ & 394 \\
\hline & Entre 21-40 & $42,97 \%$ & 1436 \\
\hline & Entre 41-60 & $42,16 \%$ & 1409 \\
\hline & Más de 61 & $3,08 \%$ & 103 \\
\hline \multirow{2}{*}{ Sexo } & Hombre & $10,02 \%$ & 335 \\
\hline & Mujer & $89,98 \%$ & 3008 \\
\hline \multirow{3}{*}{ Nivel de estudios } & Elementales & $4,01 \%$ & 134 \\
\hline & Medios & $26,94 \%$ & 900 \\
\hline & Superiores & $69,05 \%$ & 2307 \\
\hline \multirow{3}{*}{ Apoyo entorno } & Bueno & $28,11 \%$ & 939 \\
\hline & Regular & $44,06 \%$ & 1472 \\
\hline & Malo & $27,84 \%$ & 930 \\
\hline \multirow{8}{*}{ País de residencia } & España & $31,13 \%$ & 1039 \\
\hline & Italia & $8,36 \%$ & 279 \\
\hline & Francia & $2,61 \%$ & 87 \\
\hline & Portugal & $3,95 \%$ & 132 \\
\hline & Irlanda & $6,65 \%$ & 222 \\
\hline & Reino Unido & $8,96 \%$ & 299 \\
\hline & Alemania & $21,09 \%$ & 704 \\
\hline & Otro país de la UE & $17,26 \%$ & 576 \\
\hline \multirow{5}{*}{ Localidad-tamaño } & Hasta 500 habitantes & $4,08 \%$ & 136 \\
\hline & Desde 500-10.000 habitantes & $23,43 \%$ & 782 \\
\hline & Desde 10.000-250.000 habitantes & $37,49 \%$ & 1251 \\
\hline & Desde 250.000-1 millón de habitantes & $13,52 \%$ & 451 \\
\hline & Más de un millón de habitantes & $21,49 \%$ & 717 \\
\hline \multirow{2}{*}{ Ámbito de residencia } & Rural (pueblo) & $31,37 \%$ & 1048 \\
\hline & Urbano (capital) & $68,63 \%$ & 2293 \\
\hline
\end{tabular}


TABLA II

TRATAMIENTO SINTOMÁTICO UTILIZADO EN LAS CRISIS DE MIGRAÑA Y VARIABLES RELACIONADAS

\begin{tabular}{|c|c|c|c|c|c|c|c|c|c|c|c|c|c|c|}
\hline \multicolumn{15}{|c|}{ TRATAMIENTO PARA EL DOLOR } \\
\hline \multirow{2}{*}{$\begin{array}{c}\text { Variable } \\
\text { Edad }\end{array}$} & \multicolumn{2}{|c|}{$\begin{array}{l}\text { Analgésicos } \\
\text { simples }\end{array}$} & \multicolumn{2}{|c|}{ Antinflamatorios } & \multicolumn{2}{|c|}{ Triptanes } & \multicolumn{2}{|c|}{$\begin{array}{c}\text { Otros } \\
\text { tratamientos } \\
\text { sintomáticos }\end{array}$} & \multicolumn{2}{|c|}{$\begin{array}{c}\text { Varios } \\
\text { sintomáticos }\end{array}$} & \multicolumn{2}{|c|}{$\begin{array}{c}\text { No llevo } \\
\text { tratamiento }\end{array}$} & \multicolumn{2}{|c|}{$\begin{array}{c}\text { No sé qué es } \\
\text { un tratamiento } \\
\text { sintomático }\end{array}$} \\
\hline & $n$ & $\%$ & $n$ & $\%$ & $n$ & $\%$ & $n$ & $\%$ & $n$ & $\%$ & $n$ & $\%$ & $n$ & $\%$ \\
\hline Menos de 20 años & 192 & 14,7 & 168 & 13,01 & 44 & 2,31 & 38 & 7,35 & 24 & 9,2 & 43 & 32,82 & 44 & 30,77 \\
\hline Entre 21-40 & 618 & 47,32 & 614 & 47,56 & 776 & 40,82 & 250 & 48,36 & 119 & 45,59 & 51 & 38,93 & 60 & 41,96 \\
\hline Entre 41-60 & 467 & 35,76 & 483 & 37,41 & 1016 & 53,45 & 211 & 40,81 & 112 & 42,91 & 32 & 24,43 & 38 & 26,57 \\
\hline Más de 61 & 29 & 2,22 & 26 & 2,01 & 65 & 3,42 & 18 & 3,48 & 6 & 2,3 & 5 & 3,82 & 1 & 0,7 \\
\hline Total & 1306 & 100 & 1291 & 100 & 1901 & 100 & 517 & 100 & 261 & 100 & 131 & 100 & 143 & 100 \\
\hline No disponible & 4 & & 5 & & 2 & & 0 & & 0 & & 1 & & 1 & \\
\hline Género & $n$ & $\%$ & $n$ & $\%$ & $n$ & $\%$ & $n$ & $\%$ & $n$ & $\%$ & $n$ & $\%$ & $n$ & $\%$ \\
\hline Hombre & 134 & 10,24 & 126 & 9,74 & 153 & 8,05 & 39 & 7,54 & 24 & 9,2 & 23 & 17,42 & 22 & 15,28 \\
\hline Mujer & 1174 & 89,76 & 1167 & 90,26 & 1747 & 91,95 & 478 & 92,46 & 237 & 90,8 & 109 & 82,58 & 122 & 84,72 \\
\hline Total & 1308 & 100 & 1293 & 100 & 1900 & 100 & 517 & 100 & 261 & 100 & 132 & 100 & 144 & 100 \\
\hline No disponible & 2 & & 3 & & 3 & & 0 & & 0 & & 0 & & 0 & \\
\hline País & $n$ & $\%$ & $n$ & $\%$ & $n$ & $\%$ & $n$ & $\%$ & $n$ & $\%$ & $n$ & $\%$ & $n$ & $\%$ \\
\hline España & 411 & 31,45 & 504 & 39,01 & 348 & 18,32 & 117 & 22,76 & 83 & 32,05 & 70 & 53,03 & 69 & 47,92 \\
\hline Italia & 72 & 5,51 & 149 & 11,53 & 178 & 9,37 & 43 & 8,37 & 20 & 7,72 & 4 & 3,03 & 2 & 1,39 \\
\hline Francia & 22 & 1,68 & 47 & 3,64 & 62 & 3,26 & 15 & 2,92 & 8 & 3,09 & 0 & 0 & 7 & 4,86 \\
\hline Portugal & 51 & 3,9 & 72 & 5,57 & 60 & 3,16 & 33 & 6,42 & 7 & 2,7 & 1 & 0,76 & 2 & 1,39 \\
\hline Irlanda & 83 & 6,35 & 118 & 9,13 & 106 & 5,58 & 47 & 9,14 & 24 & 9,27 & 8 & 6,06 & 22 & 15,28 \\
\hline Reino Unido & 115 & 8,8 & 127 & 9,83 & 208 & 10,95 & 60 & 11,67 & 37 & 14,29 & 14 & 10,61 & 19 & 13,19 \\
\hline Alemania & 341 & 26,09 & 130 & 10,06 & 489 & 25,74 & 129 & 25,1 & 43 & 16,6 & 23 & 17,42 & 10 & 6,94 \\
\hline Otro País de la UE & 212 & 16,22 & 145 & 11,22 & 449 & 23,63 & 70 & 13,62 & 37 & 14,29 & 12 & 9,09 & 13 & 9,03 \\
\hline Total & 1307 & 100 & 1292 & 100 & 1900 & 100 & 514 & 100 & 259 & 100 & 132 & 100 & 144 & 100 \\
\hline No disponible & 3 & & 4 & & 3 & & 3 & & 2 & & 0 & & 0 & \\
\hline $\begin{array}{r}\text { Caracterí } \\
\text { de Local }\end{array}$ & $n$ & $\%$ & $n$ & $\%$ & $n$ & $\%$ & $n$ & $\%$ & $n$ & $\%$ & $n$ & $\%$ & $n$ & $\%$ \\
\hline$<500$ hab. & 50 & 3,82 & 49 & 3,8 & 78 & 4,12 & 29 & 5,62 & 16 & 6,18 & 5 & 3,79 & 7 & 4,86 \\
\hline $500-10.000$ ha & 299 & 22,86 & 292 & 22,62 & 414 & 21,85 & 110 & 21,32 & 59 & 22,78 & 40 & 30,3 & 42 & 29,17 \\
\hline 10.000-250.000 hab & 497 & 38 & 503 & 38,96 & 699 & 36,89 & 193 & 37,4 & 107 & 41,31 & 51 & 38,64 & 47 & 32,64 \\
\hline $\begin{array}{l}250.000-1 \text { millón de } \\
\text { hab }\end{array}$ & 193 & 14,76 & 190 & 14,72 & 259 & 13,67 & 70 & 13,57 & 23 & 8,88 & 14 & 10,61 & 24 & 16,67 \\
\hline >1 millón de hab. & 69 & 20,57 & 257 & 19,91 & 445 & 23,48 & 114 & 22,09 & 54 & 20,85 & 22 & 16,67 & 24 & 16,67 \\
\hline Total & 1308 & 100 & 1291 & 100 & 1895 & 100 & 516 & 100 & 259 & 100 & 132 & 100 & 144 & 100 \\
\hline No disponible & 2 & & 5 & & 8 & & 1 & & 2 & & 0 & & 0 & \\
\hline Nivel de es & $n$ & $\%$ & $n$ & $\%$ & $n$ & $\%$ & $n$ & $\%$ & $n$ & $\%$ & $n$ & $\%$ & $n$ & $\%$ \\
\hline Elementales & 57 & 4,35 & 47 & 3,63 & 52 & 2,74 & 16 & 3,1 & 10 & 3,83 & 11 & 8,33 & 13 & 9,09 \\
\hline Medios & 342 & 26,13 & 309 & 23,9 & 446 & 23,51 & 130 & 25,19 & 84 & 32,18 & 49 & 37,12 & 61 & 42,66 \\
\hline Superiores & 910 & 69,52 & 937 & 72,47 & 1399 & 73,75 & 370 & 71,71 & 167 & 63,98 & 72 & 54,55 & 69 & 48,25 \\
\hline Total & 1309 & 100 & 1293 & 100 & 1897 & 100 & 516 & 100 & 261 & 100 & 132 & 100 & 143 & 100 \\
\hline No disponible & 1 & & 3 & & 6 & & 1 & & 0 & & 0 & & 1 & \\
\hline Ámbito en el que vive & $n$ & $\%$ & $n$ & $\%$ & $n$ & $\%$ & $n$ & $\%$ & $n$ & $\%$ & $n$ & $\%$ & $n$ & $\%$ \\
\hline Rural (Pueblo) & 404 & 30,86 & 369 & 28,54 & 594 & 31,33 & 162 & 31,46 & 104 & 39,85 & 41 & 31,06 & 54 & 37,5 \\
\hline Urbano (Capital) & 905 & 69,14 & 924 & 71,46 & 1302 & 68,67 & 353 & 68,54 & 157 & 60,15 & 91 & 68,94 & 90 & 62,5 \\
\hline Total & 1309 & 100 & 1293 & 100 & 1896 & 100 & 515 & 100 & 261 & 100 & 132 & 100 & 144 & 100 \\
\hline No disponible & 1 & & 3 & & 7 & & 2 & & 0 & & 0 & & 0 & \\
\hline
\end{tabular}


TABLA III

USO DE ANALGÁSICOS SIMPLES COMO TRATAMIENTO SINTOMÁTICO PARA LAS CRISIS DE MIGRAÑA Y VARIABLES CON SIGNIFICACIÓN ESTADISTICA*

\begin{tabular}{|c|c|c|c|c|c|}
\hline Variable & \multicolumn{5}{|c|}{ TRATAMIENTO PARA EL DOLOR CON ANALGÉSICOS SIMPLES } \\
\hline \multirow{2}{*}{ Edad } & \multicolumn{2}{|c|}{ No } & \multicolumn{2}{|c|}{ Sí } & \multirow{2}{*}{ p-valor } \\
\hline & $n$ & $\%$ & $n$ & $\%$ & \\
\hline Menos de 20 años & 202 & 9,92 & 192 & 14,7 & \multirow{6}{*}{$<0,0001$} \\
\hline Entre 21-40 & 818 & 40,18 & 618 & 14,7 & \\
\hline Entre 41-60 & 942 & 46,27 & 467 & 35,76 & \\
\hline Más de 61 & 74 & 3,63 & 29 & 2,22 & \\
\hline Total & 2036 & 100 & 1306 & 100 & \\
\hline No disponible & 4 & & 4 & & \\
\hline \multirow{2}{*}{ País } & \multicolumn{2}{|c|}{ No } & \multicolumn{2}{|c|}{ Sí } & \multirow{12}{*}{$<0,0001$} \\
\hline & $n$ & $\%$ & $n$ & $\%$ & \\
\hline España & 628 & 30,92 & 411 & 31,45 & \\
\hline Italia & 207 & 10,19 & 72 & 5,51 & \\
\hline Francia & 65 & 3,2 & 22 & 1,68 & \\
\hline Portugal & 81 & 3,99 & 51 & 3,9 & \\
\hline Irlanda & 139 & 6,84 & 8 & 6,35 & \\
\hline Reino Unido & 184 & 9.06 & 115 & 8.8 & \\
\hline Alemania & 363 & 17.87 & 341 & 26.09 & \\
\hline Otro País de la UE & 364 & 17.92 & 212 & 16.22 & \\
\hline Total & 2031 & 100 & 130 & 100 & \\
\hline No disponible & 9 & & 3 & & \\
\hline
\end{tabular}

*Se incluyen solo las variables con p-valor < 0,05. Se han descartado: género, nivel de estudios, características de la localidad, ámbito de residencia por p-valor > 0,05

correspondiendo el mínimo uso de estos fármacos a los mayores de 61 años ( $p<0,0001$ ). España y Alemania son los países que mayor uso hacen de analgésicos simples ( $p<0,0001$ ) (Tabla III).

- El uso de AINE está más extendido y se corresponde con las edades comprendidas entre los 21-60 años ( $p<0,0001$ ). España, Italia y Alemania son los países que mayor uso hacen de AINE ( $p<0,0001$ ) (Tabla IV). Los pacientes con estudios superiores son los mayores consumidores de estos fármacos ( $p<0,003$ ).

- El uso de triptanes como tratamiento de las crisis muestra relación significativa con la edad, con mayor uso entre pacientes de entre 21-60 años ( $p<0,0001$ ), más en las mujeres ( $p<0,0001$ ). El mayor consumo de estos fármacos se da en Alemania, resto de países de Unión Europea, España y Reino Unido ( $p<0,0001)$, con mayor consumo en pacientes que viven en ciudades de más de 500 habitantes aunque con diferencias poco significativas $(p<0,010)$ y en pacientes con estudios medios o superiores ( $p<0,0001$ ) [Tabla V). También en este grupo de estudios medios y superiores se encuentra por contraste el mayor porcentaje de pacientes que desconocen qué es un tratamiento sintomático para las crisis de migraña ( $<<0,0001)$ (Tabla VI).

\section{DISCUSIÓN}

Las recomendaciones en lo que se refiere a criterios diagnósticos y al tratamiento de la migraña son actualizadas de forma regular por la International Headache Society (IHS), siendo la más reciente de 2018 (5). En base a ellas, la Sociedad Española de Neurología [6] especifica que el tratamiento supresor de las crisis de migraña está indicado en todos los pacientes y que debe ser individualizado. De forma genérica se diferencian dos grupos de tratamientos para las crisis: terapias inespecificas (analgésicos simples y AINE) y específicas (triptanes-agonistas de los receptores 5HTy ergóticos].

Los AINE por vía oral son los fármacos indicados en crisis leves o moderadas (nivel de evidencia I, grado de recomendación A] y, cuando no se obtenga respuesta con estos fármacos la recomendación es de triptanes (nivel de evidencia II, III y grado de recomendación C).

En crisis severas, el tratamiento indicado son los triptanes (grado de recomendación A). 
TABLA IV

USO DE AINE COMO TRATAMIENTO SINTOMÁTICO PARA LAS CRISIS DE MIGRAÑA Y VARIABLES CON SIGNIFICACIÓN ESTADISTICA*

\begin{tabular}{|c|c|c|c|c|c|}
\hline Variable & \multicolumn{5}{|c|}{ TRATAMIENTO PARA EL DOLOR CON AINE } \\
\hline \multirow{2}{*}{ Edad } & \multicolumn{2}{|c|}{ No } & \multicolumn{2}{|c|}{ Sí } & \multirow{2}{*}{$p$-valor } \\
\hline & $n$ & $\%$ & $n$ & $\%$ & \\
\hline Menos de 20 años & 226 & 11,02 & 168 & 13,01 & \multirow{6}{*}{$<0,0001$} \\
\hline Entre 21-40 & 822 & 40,08 & 614 & 47,56 & \\
\hline Entre 41-60 & 926 & 45,15 & 483 & 37,41 & \\
\hline Más de 61 & 77 & 3,75 & 26 & 2,01 & \\
\hline Total & 2051 & 100 & 1291 & 100 & \\
\hline No disponible & 3 & & 5 & & \\
\hline \multirow{2}{*}{ País } & \multicolumn{2}{|c|}{ No } & \multicolumn{2}{|c|}{ Sí } & \multirow{12}{*}{$<0,000$} \\
\hline & $n$ & $\%$ & $n$ & $\%$ & \\
\hline España & 535 & 26,15 & 504 & 39,01 & \\
\hline Italia & 130 & 6,35 & 149 & 11,53 & \\
\hline Francia & 40 & 1,96 & 47 & 3,64 & \\
\hline Portugal & 60 & 2,93 & 72 & 5,57 & \\
\hline Irlanda & 104 & 5,08 & 118 & 9,13 & \\
\hline Reino Unido & 172 & 8,41 & 127 & 9,83 & \\
\hline Alemania & 574 & 28,05 & 130 & 10,06 & \\
\hline Otro País de la UE & 431 & 21,07 & 145 & 11,22 & \\
\hline Total & 2046 & 100 & 1292 & 100 & \\
\hline No disponible & 8 & & 4 & & \\
\hline \multirow{2}{*}{ Nivel de estudios } & \multicolumn{2}{|c|}{ No } & \multicolumn{2}{|c|}{ Sí } & \multirow{7}{*}{0,003} \\
\hline & $n$ & $\%$ & $n$ & $\%$ & \\
\hline Elementales & 87 & 4,25 & 47 & 3,63 & \\
\hline Medios & 591 & 28,86 & 309 & 23,9 & \\
\hline Superiores & 137 & 66,89 & 937 & 72,47 & \\
\hline Total & 2048 & 100 & 1293 & 100 & \\
\hline No disponible & 6 & & 3 & & \\
\hline
\end{tabular}

*Se incluyen solo las variables con p-valor < 0,05. Se han descartado: género, características de la localidad, ámbito de residencia por p-valor $>0,05$.

Si bien los criterios parecen estar claros, nuestra encuesta muestra resultados muy variables. Consideramos en la encuesta como opciones de tratamiento los analgésicos simples, AINE, triptanes y otros fármacos o combinaciones de fármacos. Los resultados revelan significación estadística en relación con la edad, así los pacientes más jóvenes hacen mayor uso de analgésicos simples y/o AINE, siendo mayores consumidores de triptanes los comprendidos entre los 41-60 años.

En lo que respecta a pacientes de más de 60 años, hay que tomar en consideración que la migraña afecta especialmente al grupo de edad comprendido entre los 21 y los 60 años, siendo menos habitual entre los que superan esta edad en los que, además, las carac- terísticas clínicas de la migraña son menos típicas, lo que dificulta el diagnóstico y el tratamiento e implica consideraciones específicas en riesgos por polimedicaciones o pluripatologías que se han de valorar [7]. Los resultados de nuestro trabajo muestran que este grupo de edad es el que menor uso hace de analgésicos, AINE y triptanes.

Los resultados relacionados con el uso de analgésicos para el dolor y sus diferencias por género solo muestran significación estadística en el uso de triptanes, que es mayor entre las mujeres.

Esta tendencia sobre el uso mayor de triptanes en mujeres parece ser coincidente en otros estudios. En 2014 se publicaron resultados en Piamonte, Italia, 
TABLA V

USO DE TRIPTANES COMO TRATAMIENTO SINTOMÁTICO PARA LAS CRISIS DE MIGRAÑA Y VARIABLES CON SIGNIFICACIÓN ESTADÍSTICA*

\begin{tabular}{|c|c|c|c|c|c|}
\hline Variable & \multicolumn{5}{|c|}{ TRATAMIENTO PARA EL DOLOR: CON TRIPTANES } \\
\hline \multirow{2}{*}{ Edad } & \multicolumn{2}{|c|}{ No } & \multicolumn{2}{|c|}{ Sí } & \multirow{2}{*}{$p$-valor } \\
\hline & $n$ & $\%$ & $n$ & $\%$ & \\
\hline Menos de 20 años & 350 & 24,29 & 44 & 2,31 & \multirow{6}{*}{$<0,000$} \\
\hline Entre 21-40 & 660 & 45,8 & 776 & 40,82 & \\
\hline Entre 41-60 & 393 & 27,27 & 1016 & 53,45 & \\
\hline Más de 61 & 38 & 2,64 & 65 & 3,42 & \\
\hline Total & 1441 & 100 & 1901 & 100 & \\
\hline No disponible & 6 & & 2 & & \\
\hline \multirow{2}{*}{ Género } & \multicolumn{2}{|c|}{ No } & \multicolumn{2}{|c|}{ Sí } & \multirow{6}{*}{$<0,000$} \\
\hline & $n$ & $\%$ & $n$ & $\%$ & \\
\hline Hombre & 182 & 12,61 & 153 & 8,05 & \\
\hline Mujer & 126 & 87,39 & 1747 & 91,95 & \\
\hline Total & 1443 & 100 & 1900 & 100 & \\
\hline No disponible & 4 & & 3 & & \\
\hline \multirow{2}{*}{ País } & \multicolumn{2}{|c|}{ No } & \multicolumn{2}{|c|}{ Sí } & \multirow{12}{*}{$<0,0001$} \\
\hline & $n$ & $\%$ & $n$ & $\%$ & \\
\hline España & 691 & 48,05 & 348 & 18,32 & \\
\hline Italia & 101 & 7,02 & 178 & 9,37 & \\
\hline Francia & 25 & 1,74 & 62 & 3,26 & \\
\hline Portugal & 72 & 5,01 & 60 & 3,16 & \\
\hline Irlanda & 116 & 8,07 & 106 & 5,58 & \\
\hline Reino Unido & 91 & 6,33 & 208 & 10,95 & \\
\hline Alemania & 215 & 14,95 & 489 & 25,74 & \\
\hline Otro País de la UE & 127 & 8,83 & 449 & 23,63 & \\
\hline Total & 1438 & 100 & 1900 & 100 & \\
\hline No disponible & 9 & & 3 & & \\
\hline \multirow{2}{*}{ Características localidad de residencia } & \multicolumn{2}{|c|}{ No } & \multicolumn{2}{|c|}{ Sí } & \multirow{9}{*}{0,010} \\
\hline & $n$ & $\%$ & $n$ & $\%$ & \\
\hline Hasta 500 habitantes & 58 & 4,02 & 78 & 4,12 & \\
\hline Desde 500-10.000 habitantes & 368 & 25,52 & 414 & 21,85 & \\
\hline Desde 10.000-250.000 habitantes & 552 & 38,28 & 699 & 36,89 & \\
\hline Desde 250.000-1 millón de habitantes & 192 & 13,31 & 259 & 13,67 & \\
\hline Más de 1 millón de habitantes & 272 & 18,86 & 445 & 23,48 & \\
\hline Total & 1442 & 100 & 1895 & 100 & \\
\hline No disponible & 5 & & 8 & & \\
\hline \multirow{2}{*}{ Nivel de estudios } & \multicolumn{2}{|c|}{ No } & \multicolumn{2}{|c|}{ Sí } & \multirow{7}{*}{$<0,0001$} \\
\hline & $n$ & $\%$ & $n$ & $\%$ & \\
\hline Elementales & 82 & 5,68 & 52 & 2,74 & \\
\hline Medios & 454 & 31,44 & 446 & 23,51 & \\
\hline Superiores & 908 & 62,88 & 1399 & 73,75 & \\
\hline Total & 144 & 100 & 1897 & 100 & \\
\hline No disponible & 3 & & 6 & & \\
\hline
\end{tabular}

*Se incluyen solo las variables con p-valor < 0,05. Se han descartado: ámbito de residencia por p-valor > 0,05. 
TABLA VI

DESCONOCIMIENTO DE TRATAMIENTO SINTOMÁTICO PARA LAS CRISIS DE MIGRAÑA Y VARIABLES CON SIGNIFICACIÓN ESTADÍSTICA*

\begin{tabular}{|c|c|c|c|c|c|}
\hline Variable & \multicolumn{5}{|c|}{$\begin{array}{c}\text { TRATAMIENTO PARA EL DOLOR, NO SÉ QUÉ ES UN } \\
\text { TRATAMIENTO SINTOMÁTICO }\end{array}$} \\
\hline \multirow{2}{*}{ Nivel de estudios } & \multicolumn{2}{|c|}{ No } & \multicolumn{2}{|c|}{ Si } & $p$-valor \\
\hline & $n$ & $\%$ & $n$ & $\%$ & \multirow{6}{*}{$<0,0001$} \\
\hline Elementales & 121 & 3,78 & 13 & 9,09 & \\
\hline Medios & 839 & 26,24 & 61 & 42,66 & \\
\hline Superiores & 2238 & 69,98 & 69 & 48,25 & \\
\hline Total & 3198 & 100 & 143 & 100 & \\
\hline No disponible & 8 & & 1 & & \\
\hline
\end{tabular}

*Se incluyen solo las variables con p-valor < 0,05. Se han descartado: edad, género, ámbito de residencia país, características de la localidad, ámbito de residencia por p-valor > 0,05.

sobre los patrones de uso y administración de medicamentos en pacientes que solicitan asistencia de farmacéuticos para aliviar un ataque de migraña. Todos los estudios epidemiológicos sobre la migraña han demostrado sistemáticamente que es mucho más común entre las mujeres que entre los hombres. Esta diferencia de género también se refleja en el mayor porcentaje de mujeres que reciben tratamiento o consejos para los ataques de dolor de cabeza. Entre los fármacos que generalmente se toman para aliviar el dolor de cabeza, no hubo diferencias estadísticamente significativas entre hombres y mujeres en el uso habitual de AINE, al igual que los resultados obtenidos en nuestro trabajo. Las diferencias estadísticamente significativas surgieron en el uso de triptanes y en el uso de medicamentos combinados, pero no en el uso de analgésicos simples [8].

En nuestra encuesta se observan diferencias por países en el uso de tratamientos para el dolor. España y Reino Unido son los que menos recurren a tratamientos y, junto con Francia e Irlanda, son los que mayor desconocimiento muestran en tratamiento sintomático. Irlanda Reino Unido y Alemania son los que más recurren a otros tratamientos, siendo los triptanes los más utilizados en todos los países excepto en España, Portugal e Irlanda. Los AINE son usados en todos los países participantes en la encuesta, menos en Alemania y en el grupo de otros países de la unión europea, y los analgésicos simples son de uso prioritario en España y Alemania. Los resultados coinciden con lo comentado previamente en el estudio Eurolight previamente comentado, si bien en este trabajo no consta la diferenciación por grupos farmacológicos que se ha hecho en nuestro estudio.

En nuestro trabajo, el uso de tratamientos sintomáticos no se ve influido por el número de habitantes de la localidad, pero el nivel de estudios sí condiciona el tratamiento sintomático utilizado, siendo mayor el de AINE y triptanes en personas con estudios superiores y, por el contrario, el desconocimiento de los tratamientos o no uso de los mismos predomina en pacientes con estudios elementales o medios.

Independientemente del nivel formativo de los pacientes, parece haber consenso en la comunidad científica sobre las ventajas de disponer de formación e infor- mación sobre la patología. Los pacientes consideran que disponer de información básica como un folleto educativo sobre la migraña comprensible, supone un medio para aumentar el conocimiento general de la enfermedad y resulta de utilidad para aumentar el control sobre las crisis [9].

En nuestro trabajo el ámbito de residencia parece mostrar una tendencia al uso de AINE en zonas urbanas y al uso de otros tratamientos en zonas rurales. En la mayor parte de los países las barreras para buscar o acceder a atención médica en poblaciones rurales es mayor que en las zonas urbanas, especialmente en poblaciones pequeñas y alejadas de los núcleos urbanos. Las revisiones bibliográficas realizadas muestran en países como Estados Unidos disparidades en la atención de salud rural y urbana y orientan hacia programas continuos de reforma con el objetivo de mejorar la provisión de servicios sanitarios, promover el reclutamiento, la capacitación y el desarrollo profesional de los sanitarios responsables de la atención de salud rural, aumentar la cobertura de seguro de salud integral e involucrar a residentes rurales y proveedores de atención médica en la promoción de la salud [10]. Este aspecto coincide con lo observado en países procedentes de áreas socioculturales muy diversas y que incluyen aspectos ligados a deficiencias en el acceso a la atención médica y de enfermería (11).

Los resultados de nuestro estudio revelan una gran variabilidad de los resultados en cada país y que se modifican en función de las distintas condiciones sociales y demográficas. Estos resultados son concordantes con la línea planteada por otros autores en que esta variabilidad facilita la evolución hacia procesos crónicos de migraña y que todas las clases de medicamentos pueden inducir esta cronificación de la patología. Los medicamentos que tienen un mayor riesgo de uso excesivo se encuentran entre los preferidos por los pacientes más difíciles de tratar por su mala respuesta y por un impulso particular hacia el consumo de "todo lo que pueda percibirse para brindar algún alivio" y, a pesar de que estos medicamentos que se perciben como "más potentes", a menudo son los que se indican como medicamentos de segunda o tercera línea (12). 
Las recomendaciones de tratamiento para la crisis de migraña se basan como punto de partida en el correcto diagnóstico, atendiendo a los criterios de consenso y con terapias aplicadas de forma individualizada según las condiciones de cada paciente. Hay varios medicamentos disponibles para el tratamiento de la migraña aguda, pero no todos son efectivos para todos los pacientes, ni igualmente efectivos en todos los ataques. Actualmente, el grupo de fármacos agonistas de la serotonina (5-HT) 1B/1D, los denominados triptanes, constituye el pilar de los regímenes terapéuticos agudos, si bien existen otros enfoques para el tratamiento agudo, como analgésicos simples, medicamentos antinflamatorios no esteroideos (AINE), ergotamínicos y medicamentos combinados. Actualmente se valoran otros tratamientos más recientes, ya en su uso o bajo investigación clínica (13).

Los perfiles de seguridad de los medicamentos para la migraña limitan su uso en pacientes con ciertas condiciones comórbidas, y los efectos adversos también pueden reducir el nivel de cumplimiento del paciente. Los AINE se asocian frecuentemente con efectos secundarios gastrointestinales y posiblemente también cardiovasculares. Los alcaloides ergóticos pueden inducir vasoconstricción arterial, mientras que la administración de triptanes está contraindicada en enfermedades cardiovasculares, cerebrovasculares y vasculares periféricas. Una serie de fármacos experimentales recientemente sintetizados parecen ser efectivos y prometedores para el tratamiento de la migraña, pero en la actualidad la experiencia con ellos aún es limitada, por lo que es esencial realizar más estudios (14).

En el momento actual se discuten los diferentes tipos de medicamentos para la migraña aguda, con especial atención a los problemas de seguridad y los posibles efectos adversos. Aunque los triptanes se usan ampliamente en el tratamiento agudo de la migraña, existe incertidumbre acerca de la eficacia comparativa entre sí y frente a los otros tratamientos específicos o usados en migraña. Los triptanes utilizados a dosis estándar se asocian con mejores resultados que los ergotamínicos y con resultados iguales o mejores en comparación con los AINE, salicilatos y analgésicos simples [15). El uso frecuente de analgésicos, alcaloides de ergotamínicos y triptanes puede repercutir en el desarrollo de dolor de cabeza crónico por uso excesivo de medicamentos; a esto se añade el hecho de que el inicio de un ataque de migraña no se comprende completamente, y el tratamiento dirigido a los factores causales no está disponible actualmente. La tolerabilidad y los efectos adversos de los medicamentos disponibles en la actualidad limitan su utilización en determinados grupos de pacientes, junto con el hecho de que el uso frecuente de estos medicamentos plantea riesgo de desarrollar efectos adversos y, por ello, se impone la necesidad de medicamentos basados en mecanismos patológicos dentro de un concepto de medicina personalizada (16).

\section{AGRADECIMIENTOS}

A los pacientes de la EMHA por su colaboración voluntaria en esta encuesta, a la Asociación Española de Especialistas en Medicina del Trabajo por su apoyo y aval científico, a Silvia Lladosa por el estudio estadístico de los datos.

\section{CONFLICTO DE INTERESES}

Este trabajo ha contado con financiación de la European Migraine \& Headache Alliance.

\section{ANEXO 1 \\ CUESTIONARIO MIGRAÑA Y TRABAJO. ESTUDIO DE SITUACIÓN}

Tiempo aproximado para responder esta encuesta: 6 minutos
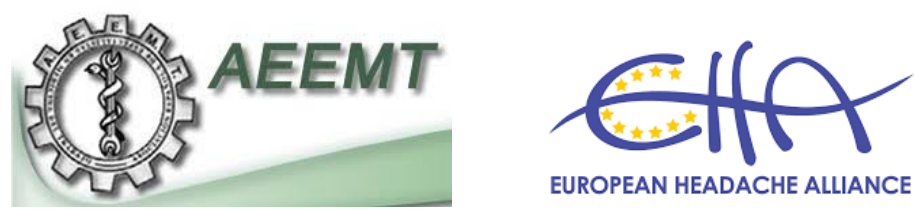

EUROPEAN HEADACHE ALLIANCE

El objetivo de este estudio es conocer la situación del trabajador con migraña dentro del mundo del trabajo y comparar la situación en los distintos países participantes.

De esta forma, y con los datos resultantes, se puede favorecer la puesta en marcha de actuaciones para mejorar el entorno laboral y la máxima integración del trabajador con migraña dentro de las empresas.

Se desea implementar las medidas preventivas y adaptativas con beneficio común para: el trabajador, el sanitario y el empresario.

*Esta encuesta se ha llevado acabo con el aval científico de la Asociación Española de Especialistas en Medicina del Trabajo (AEEMT). 


\section{COMPRUEBE QUE CUMPLE LOS CRITERIOS DE INCLUSIÓN PARA EL ESTUDIO}

Su participación es voluntaria

Conoce que los datos que se obtengan serán de uso confidencial

Cumple los criterios de migraña

Está trabajando en el momento de la encuesta, o lo ha estado en el año previo

\section{CUESTIONES DEL PACIENTE/TRABAJADOR: RELATIVAS A SUS DATOS PERSONALES}

1. Edad:

- Menos de 20 años

- Entre 21-40 años

- Entre 41-60 años

- Más de 61 años

2. Sexo:

- Hombre

- Mujer

3. Lugar de residencia:

- España

- Italia

- Francia

- Portugal

- Irlanda

- Reino Unido

- Alemania

- Otro país de la UE

4. Características de su localidad de residencia: número aproximado de habitantes:

- Hasta 500 habitantes

- Desde 500-10.000 habitantes

- Desde 10.000-250.000 habitantes

- Desde 250.000-1 millón de habitantes

- Más de un millón de habitantes

5. Nivel de estudios:

- Elementales

- Medios

- Superiores

6. Apoyo que recibe el trabajador de su entorno durante las crisis de migraña:

- Bueno

- Regultar

- Malo

7. Ámbito en el que vive:

- Rural (pueblo)

- Urbano (capital)

\section{CUESTIONES DEL PACIENTE/TRABAJADOR: RELATIVAS A SU MIGRAÑA}

8. Tipo de migraña que padece:

- Con aura

- Sin aura

- Ambos tipos

- Crónica/Cronificada

9. Duración de las crisis:

- Menos de 4 horas

- Entre 4-6 horas

- Más de 6 horas 
10. Frecuencia de las crisis:

- Menos de 3/mes

- Entre 3-6/mes

- Más de 6/mes

11. Control médico (MARCAR LAS OPCIONES NECESARIAS, PUEDEN SER MÁS DE UNA):

- Por neurólogo

- Por médico general/de familia/Atención Primaria

- Por médico del trabajo

- Por otro médico/otras especialidades

- Por enfermería

- No llevo control médico/autocontrol

12. Tratamiento preventivo de las crisis de migraña (MARCAR LAS OPCIONES NECESARIAS, PUEDEN SER MÁS DE UNA):

- Llevo un tratamiento preventivo siempre

- Llevo un tratamiento preventivo en temporadas

- Llevo varios tratamientos preventivos siempre

- Llevo varios tratamientos preventivos en temporadas

- No llevo tratamiento preventivo

- No sé qué es un tratamiento preventivo

13. Tratamiento para el dolor cuando tiene crisis de migraña (MARCAR LAS OPCIONES NECESARIAS, PUEDEN SER MÁS DE UNA):

- Con analgésicos simples

- Con antinflamatorios

- Con triptanes

- Con otros tratamientos sintomáticos

- Con varios tratamientos sintomáticos no nombrados anteriormente

- No llevo tratamientos sintomáticos

- No sé qué es un tratamiento sintomático

14. ¿Usa otros tratamientos complementarios (dietas, fisioterapia, mindfulness, etc.?:

- Sí

- No

\section{CUESTIONES DEL PACIENTE/TRABAJADOR: RELATIVAS A SU TRABAJO}

15. Empresa/Sector del trabajo actual del trabajador:

- Autónomo/por cuenta propia

- Por cuenta ajena:

- Construcción

- Industria

- Sanitario

- Hostelería

- Administración pública

- Servicios comerciales

- Otros servicios: abogado, ingeniero, arquitecto, consultor, asesor

- Docencia

- Otros sectores profesionales

16. Puesto de trabajo actual del trabajador:

- Administrativo

- Comercial

- Limpieza

- Mantenimiento

- Fuerzas del orden público

- Sanitario

- Operario de la industria

- Dependiente/Atención al cliente

- Mando intermedio

- Puesto directivo

- Profesor

- Otros 
17. Riesgos del puesto que desempeña (MARCAR LAS OPCIONES NECESARIAS, PUEDEN SER MÁS DE UNA):

- Manipulación de cargas

- Exposición al ruido

- Exposición a sustancias químicas

- Estrés laboral

- Turnos de trabajo rotatorios o nocturnos

- Conducción de vehículos (más de 1/3 de la jornada laboral)

- Vibraciones

- Trabajos que requieren gran atención o de precisión

- Manejo de maquinaria de riesgo (carretillas elevadoras o similares]

- Malas condiciones medioambientales (temperatura, humedad...)

- Ergonomía inadecuada (mobiliario y herramientas o utensilios de trabajo no adecuados)

- Uso de pantallas de visualización de datos/ordenadores

- Otros

- Desconozco los riesgos del puesto

18. Tamaño de la empresa donde trabaja:

- Microempresa (con menos de 10 trabajadores)

- Pequeña empresa (entre 11 y 49 trabajadores)

- Mediana empresa (entre 50 y 250 trabajadores)

- Empresa grande (más de 250 trabajadores)

19. Ubicación de la empresa donde trabaja:

- Urbano (capital o polígono de la capital)

- Rural (pueblo o polígono industrial aislado)

20. Servicio de prevención en la empresa donde trabaja:

- Propio (de la empresa)

- Ajeno (concertado con otra empresa)

- Desconozco el tipo de servicio de prevención

21. Servicio médico en la empresa en la que trabaja:

- Sí hay, a jornada completa

- Sí hay, a tiempo parcial

- No se dispone en la empresa

- Desconozco si existe servicio médico en la empresa

22. Reconocimientos periódicos de vigilancia de la salud en la empresa que trabaja:

- Sí, voy todos los años

- Sí, voy cada dos años

- Sí, voy esporádicamente

- No voy nunca

- Desconozco si hay o acude a los reconocimientos de vigilancia de la salud

23. Opciones de gestión de la empresa:

- ¿La migraña le ha impedido acceder a algún trabajo?:

- Sí

- No

- ¿Le han despedido del trabajo o no renovado su contrato por padecer migraña?:

- Sí

- No

- ¿Ha tenido dificultades en su empresa por padecer migraña (amonestaciones, sanción por bajo rendimiento, ausencias del trabajo o dudas sobre mis ausencias del trabajo por crisis de migraña...)?:

- Sí

- No

- En el caso de que haya tenido dificultades o conflicto laboral por limitaciones-pérdida de productividad para realizar adecuadamente su trabajo por padecer migraña, ¿con qué frecuencia?:

- Diariamente

- Semanalmente

- Alguna vez al mes

- De forma muy esporádica

- No repercute en mi trabajo

- ¿Ha solicitado ser considerado como trabajador especialmente sensible por su migraña con relación

al puesto de trabajo que desempeña? (en España art. 25 LPRL):

- Sí

- No

- No sé lo que es eso 
- ¿Ha solicitado alguna vez modificación de sus condiciones de trabajo por la migraña (de lugar, horario, funciones asignadas, etc.)?:

- Sí

- No

- ¿Ha solicitado alguna vez cambio de puesto de trabajo por la migraña?:

- Sí

- No

- Si lo ha solicitado, ¿le han adaptado o ajustado de alguna forma el puesto de trabajo en su empresa por la migraña (cambio de puesto o de lugar, horario, funciones asignadas, etc.)?:

- Sí

- No

- ¿Se ha sentido comprendido o apoyado por su empresa por las limitaciones que implica la migraก๊a?:

- Sí

- No

- ¿Se ha sentido comprendido y apoyado por sus compañeros en relación a las limitaciones que implica la migraña?

- Sí

- No

24. Precepción personal de su capacidad laboral los días sin migraña (autopercepción):

- Los días que usted NO SUFRE UNA CRISIS DE MIGRAÑA: ¿se considera limitado para realizar adecuadamente su trabajo?:

- Sí, diariamente

- Sí, semanalmente

- Sí, alguna vez al mes

- Sí, pero de forma muy esporádica

- No repercute en mi trabajo

25. Percepción personal de su capacidad laboral los días sin migraña (autopercepción):

- Los días que NO SUFRE UNA CRISIS, ¿para qué trabajos se considera limitado por las secuelas de la migraña o sus tratamientos?:

- Para ninguno, puedo realizar cualquier trabajo

- Me considero limitado para algunos trabajos

- Me considero limitado para todos los trabajos

26. Percepción personal de su capacidad laboral os días que sufre migraña (autopercepción):

- Los días que usted SI TIENE UNA CRISIS DE MIGRAÑA, ¿Considera que la migraña puede llegar a imposibilitar la realización de su trabajo?:

- Sí

- No

27. ¿Considera que por ser afectado de migraña es usted una persona discapacitada?

- No

- Sí, pero solo durante las crisis

- Sí, todo el tiempo

28. ¿Considera que el hecho de trabajar aunque sufra migraña le facilita la integración social?:

- Sí

- No

29. ¿Considera que el mundo del trabajo facilita la integración de la persona con migraña?:

- Sí

- No

30. ¿Qué pediría a las empresas para mejorar la situación de los trabajadores que como usted sufren de migraña? (MARCAR LAS OPCIONES NECESARIAS, PUEDEN SER MÁS DE UNA):

- Flexibilidad horaria

- Opciones de adaptaciones en el puesto de trabajo

- Opciones de cambios de puesto de trabajo

- Trabajar desde casa/teletrabajo

- Tener en la empresa zonas de descanso/silencio

- Tener servicio sanitario en mi empresa (médico/enfermería)

- Varias o todas ellas

\section{GRACIAS POR SU COLABORACIÓN}




\section{BIBLIOGRAFÍA}

1. Katsarava Z, Mania M, Lampl C, Herberhold J, Steiner TJ. Poor medical care for people with migraine in Europe - evidence from the Eurolight study. J Headache Pain. 2018;19(1):10. DOl: 10.1186/s10194-018-0839-1.

2. Martelletti P, Schwedt TJ, Lanteri-Minet M, Quintana R, Carboni V, Diener HC, et al. My Migraine Voice survey: a global study of disease burden among individuals with migraine for whom preventive treatments have failed. $J$ Headache Pain. 2018;19(1):115. DOI: 10.1186/s10194-018-0946-z.

3. Vo P, Fang J, Bilitou A, Laflamme AK, Gupta S. Patients perspective on the burden of migraine in Europe: a cross-sectional analysis of survey data in France, Germany, Italy, Spain, and the United Kingdom. J Headache Pain. 2018;19(1):82. DOI: 10.1186/s10194-018-0907-6.

4. Brandes JL. Global trends in migraine care: results from the MAZE survey. CNS Drugs. 2002;16 Suppl 1:13-8. DOI: 10.2165/00023210-200216001-00003.

5. Headache Classification Committee of the International Headache Society (IHS) The International Classification of Headache Disorders, $3^{\text {rd }}$ edition. Cephalalgia. 2018;38(1):1-211. DOI: 10.1177/0333102417738202.

6. Sociedad española de neurología. Guía Oficial de práctica clínica en Cefaleas. Comité del Grupo de estudio de cefaleas SEN. 2015. Disponible en: cefaleas.sen.es/pdf/GUIA_NEURO_2015.pdf. Consultado el 13/05/2019.

7. Mathew S, Ailani J. Traditional and Novel Migraine Therapy in the Aging Population. Curr Pain Headache Rep. 2019;23(6):42. DOI: 10.1007/s11916-019-0789-6.

8. Brusa P, Allais G, Rolando S, Baratta F, Giaccone M, Bussone G, et al. Migraine attacks in the pharmacy: a gender subanalysis on treatment preferences. Neurol
Sci. 2015;36 Suppl 1:93-5. DOI: 10.1007/s10072015-2156-7.

9. Medrano Martínez V, Callejo Domínguez JM, Beltrán Lasco I, Pérez Carmona N, Abellán Miralles I, González Caballero $G$, et al. Migraine education brochures and patientperceived satisfaction. Neurologia. 2015;30(8):472-8. DOI: 10.1016/j.nrl.2014.04.010.

10. Douthit N, Kiv S, Dwolatzky T, Biswas S. Exposing some important barriers to health care access in the rural USA. Public Health. 2015;129(6):611-20. DOI: 10.1016/j. puhe.2015.04.001.

11. Lin SW, Yen CF, Chiu TY, Chi WC, Liou TH. New indices for home nursing care resource disparities in rural and urban areas, based on geocoding and geographic distance barriers: a cross-sectional study. Int J Health Geogr. 2015;14:28. DOI: 10.1186/s12942-015-0021-9.

12. Grazzi L, Grignani E, D’Amico D, Sansone E, Raggi A. Is Medication Overuse Drug Specific or Not? Data from a Review of Published Literature and from an Original Study on Italian MOH Patients. Curr Pain Headache Rep. 2018;22(11):71. DOI: 10.1007/s11916-018-0729-x.

13. Brandes JL, Buchanan TM, Welch KM. Acute treatment of migraine. Handb Clin Neurol. 2010;97:323-36. DOI: 10.1016/S0072-9752(10)97026-7.

14. Csépány É, Magyar M, Gyüre T, Bozsik G, Ertsey C. Current pharmacotherapy in migraine. Neuropsychopharmacol Hung. 2015;17(4):169-76.

15. Cameron C, Kelly S, Hsieh SC, Murphy M, Chen L, Kotb A, et al. Triptans in the Acute Treatment of Migraine: A Systematic Review and Network Meta-Analysis. Headache. 2015;55 Suppl 4:221-35. DOI: 10.1111/head.12601.

16. Tajti J, Majláth Z, SzokD, Csáti A, Vécsei L. Drug safety in acute migraine treatment. Expert Opin Drug Saf. 2015;14(6):891909. DOI: 10.1517/14740338.2015.1026325. 